\title{
Modeling Noise in Gas Cascade Secondary Electron Amplifiers
}

\author{
V. Tileli and B. L. Thiel
}

College of Nanoscale Science and Engineering, University at Albany, 255 Fuller Road, Albany NY 12203

The purpose of this work is to model the signal-to-noise ratio (SNR) of cascade amplified secondary electron signals in an Environmental SEM. In a high vacuum SEM, the emitted secondary electrons, the signal, are detected and then amplified. However, in an Environmental SEM both the desired and background signals are amplified by a gas cascade process which is then measured by the detector. Because the background consists of slowly varying component arising from backscattered and primary electrons, signal-to-background discrimination can take place later by setting the brightness (dc offset) of the image. Operating parameters such as accelerating voltage, gas pressure, cascade distance and detector bias can affect the desired and the background signals in an inter- dependent and complicated way. The statistics involved with this process are therefore more complicated than in the case of a conventional SEM and become an important factor in choosing operating conditions for optimizing image quality and SNR.

We have developed a model that takes into account beam shot, cascade and detector contributions to noise. In particular for the cascade noise, McIntyre's equations for noise in avalanche photodiodes are employed under single carrier initiated / single carrier multiplication conditions [1, 2]. This theory provides a cascade noise factor of the form: $N_{\text {cascade }}=\sqrt{M^{2} F_{e}}$ where $M$ is the amplification factor, the gain, and $F_{e}=2-1 / M$ is the excess noise factor, giving the gain uncertainty. All factors contributing to noise are incorporating into the model, secondary electrons (SE), backscattered electrons (BSE) and primary electrons (PE) by applying Moncrieff's equations for their multiplication processes [3]. The total noise is computed by summing up statistically all noise contributions.

Two different detector geometries are analyzed. The simplest case is when the anode/detector is made by biasing the final pressure limiting aperture. For this geometry, the detector is directly above the specimen and is concentric about the optic axis. This configuration generates a constant electric field with evenly spaced equipotentials (shown in Fig. 1a) is created between the specimen surface and the biased anode/detector. Secondary electrons are amplified with the ionization efficiency being more or less constant along the cascade distance. Using Townsend formulas for gas ionization efficiency, [4] the model was applied and the SNR was calculated. The operating parameters that were used for all modeling were accelerating voltage $5 \mathrm{kV}$ with a detector bias $400 \mathrm{~V}$ in water vapor. The results were plotted as a function of pressure, in Torr, for three different gas path lengths, and are shown in Fig. 1b. All curves exhibit a similar form revealing that there is an optimum for SNR which varies with operating conditions. Notice that while the pressure at which the maximum occurs changes with distance, the value of the maximum stays constant. This indicates that the product $P d$ is in fact the determining factor for optimization of SNR for this geometry.

For an off-axis anode, the geometry becomes more complicated. Figure $2 \mathrm{a}$ shows an example of this configuration. Significantly, the density of equipotentials increase with the square of distance from the sample to the detector. The electric field is no longer uniform, but increases linearly between the 
sample and detector. Consequently, the ionization efficiency changes depending on position within the cascade path. By modifying the model to fit this field geometry, the plot of SNR as a function of pressure was obtained, Fig $2 \mathrm{~b}$. The curves still have the same general form as the simple case of concentric detector geometry about the optic axis, however this time the amplitude of the maximum changes with working distance, becoming smaller with shorter working distances. This occurs because in the off-axis geometry the ionization path for backscattered and primary electrons (the main source of background) is decoupled from the cascade path. We note that off-axis detectors are typically used in a lower pressure range than the first type, so that longer cascade length is necessary to achieve acceptable gas amplification. This results in considerably lower background levels and increased signal quality.

\section{References}

[1] R.J. McIntyre, IEEE Trans. El. Dev. ED 13 (1966) 164.

[2] R.J. McIntyre, IEEE Trans. El. Dev. 46 (1999) 1623.

[3] D.A. Moncrieff et al., J.Phys. D 11 (1978) 2315.

[4] A. von Engel, Ionized Gases, Clarendon Press, Oxford, 1965.

(a)

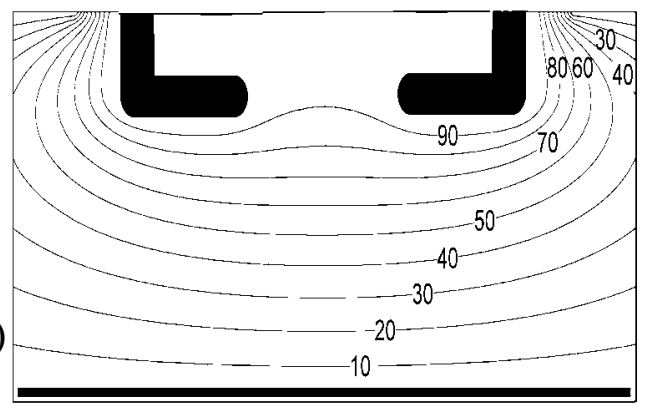

(b)

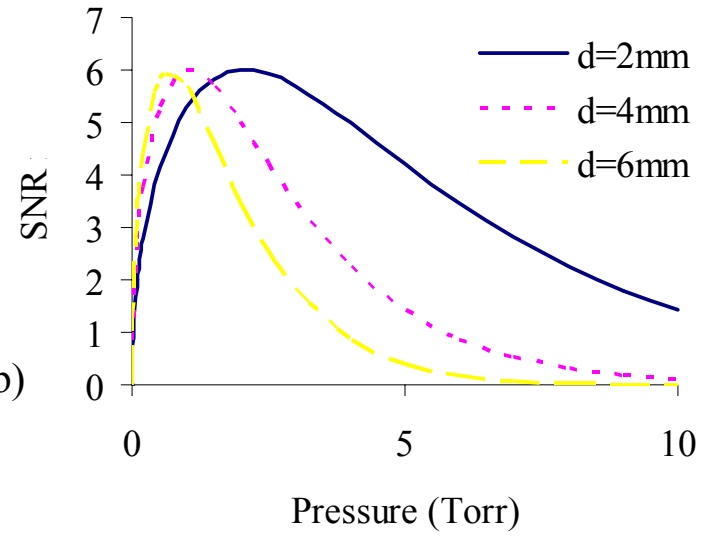

Fig. 1. Concentric about the optic axis detector geometry. (a) Equipotential lines labeled in Volts. (b) Signal-to-Noise Ratio as a function of pressure for three different gas path lengths.

(a)

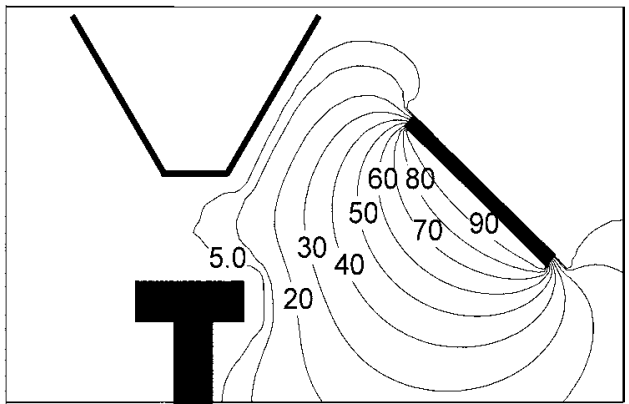

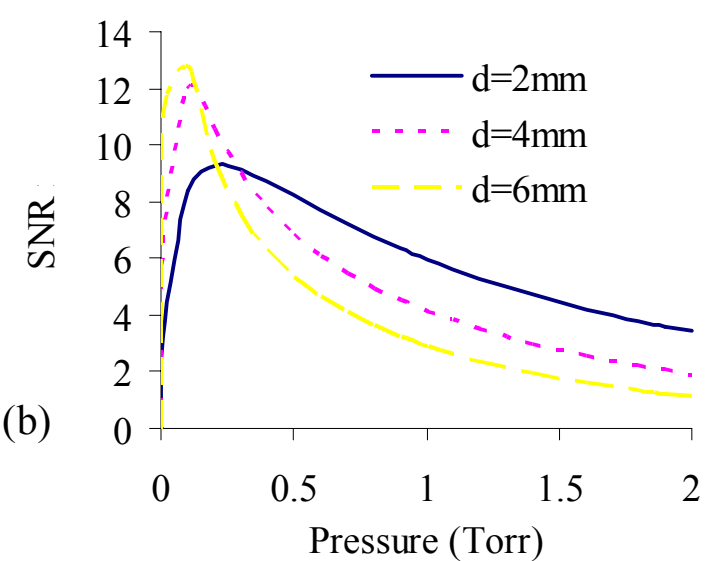

Fig. 2. Off-axis detector geometry. (a) Equipotential lines labeled in Volts. (b) Signal-to-Noise Ratio as a function of pressure for three different gas path lengths. 